\title{
Ongoing angiogenesis in blood vessels of the abdominal aortic aneurysm
}

\author{
David C. Paik ${ }^{1,4}$, Chenzhong $\mathrm{Fu}^{2}$, \\ Jahar Bhattacharya ${ }^{2}$ and M. David Tilson ${ }^{3}$ \\ ${ }^{1}$ Department of Ophthalmology and Medicine \\ College of Physicians \& Surgeons \\ Columbia University, New York, NY 10032 \\ ${ }^{2}$ Department of Medicine \\ ${ }^{3}$ Department of Surgery \\ St.Luke's/Roosevelt Hospital Center \\ College of Physicians \& Surgeons \\ Columbia University, New York, NY 10019 \\ ${ }^{4}$ Corresponding author: Tel, 212-305-1622; \\ Fax, 212-305-7238; E-mail, dcp14@columbia.edu \\ Accepted 15 November 2004
}

Abbreviations: AAA, abdominal aortic aneurysm; ECM, extracellular matrix; eNOS, endothelial nitric oxide synthase; MMP, matrix metalloproteinase

\begin{abstract}
Pathogenesis of the abdominal aortic aneurysm has been attributed to neovascularization of the aortic wall. However, it is not clear whether angiogenesis persists in the aneurysm. In sections of aneurysms, we determined the immunohistochemical distributions of the $\alpha_{v} \beta_{3}$ integrin, tenascin and endothelial nitric oxide synthase (eNOS), which are markers respectively, of angiogenesis, matrix remodeling and vasoregulatory function. In addition, we used reverse transcription followed by in situ PCR, to determine the distribution of $\alpha_{w}$ mRNA. All aneurysm specimens exhibited extensive increases of wall vascularization as compared with the control aortic wall, and showed the presence of perivascular inflammatory exudates containing macrophages and lymphocytes. The neovascularization consisted of thick-walled vessels in the media and adventitia, and capillaries in the subintima. The majority of vessels stained positively for the $\alpha_{v} \beta_{3}$ antigen and eNOS. Tenascin was deposited as bands that circumscribed thick-walled vessels. The distribution of av mRNA was extensive and was positive even in those vessels that failed to stain for the $\alpha_{v} \beta_{3}$ protein. No staining
\end{abstract}

was evident in control aortas for the $\alpha_{w} \beta_{3}$ antigen, tenascin or $\alpha_{v}$ mRNA. The upregulation of $\alpha_{v}$ mRNA and the $\alpha_{v} \beta_{3}$ integrin in blood vessels surrounded by a matrix expressing tenascin, indicates that angiogenesis is an ongoing process in the mature aortic aneurysm.

Keywords: angiogenesis; aortic aneurysm; extracellular matrix; immunohistochemistry; integrin; RT in situ PCR

\section{Introduction}

The pathophysiology of the abdominal aortic aneurysm (AAA), which carries an annual mortality in the USA of $\sim 15,000$ (Brophy et al., 1991) is not well understood; and its treatment is restricted to surgical repair with its attendant risks of morbidity and mortality. The pathophysiological understanding of the aneurysm is based largely on biochemical and immunohistochemical studies that indicate aortic wall remodelling and proteolysis of matrix proteins (Tilson et al., 1990). A multicellular inflammatory infiltrate and increased expression of tissue-type and urokinasetype plasminogen activators have also been demonstrated in the aneurysm wall (Koch et al., 1990; Brophy et al., 1991; Schneiderman et al., 1995).

A conspicuous feature of the aneurysm is neovascularization of the aortic wall, which, in contrast to normal, becomes enriched with microvessels (Koch et al., 1990; Tilson et al., 1990; Brophy et al., 1991; Holmes et al., 1995; Schneiderman et al., 1995). Although the significance of the neovascularization remains unclear, Herron et al. (1991) speculated that the new vessels play a sustaining or even a causal role in the pathophysiology of the aneurysm by secreting proteinases that destabilize the aortic matrix. Supportive evidence for this hypothesis comes from immunohistochemical studies in which proteinases such as gelatinase (matrix metalloproteinase 2, MMP2) (Herron et al., 1991) and collagenase (MMP-1) (Irizarry et al., 1993) have been localized to the neovascular endothelium.

A highly relevant but poorly understood question is whether the neovascularization is an ongoing process in well-developed aneurysms. If neovascularization were ongoing it would signify the presence of active disease in the aneurysm wall and would support the hypothesis that the new vessels are critical in the 
progression of the aneurysm. We address this question through detection of the $\alpha_{w} \beta_{3}$ integrin in adventitial vessels of aneurysms. The integrin $\alpha_{v} \beta_{3}$ has been proposed to play a critical role in angiogenesis. Brooks et al. have reported that this adhesion molecule is a marker for active angiogenic vessels in man and the chick, and that blocking its activity with a monoclonal antibody suppresses angiogenesis (Brooks et al., 1994).

During angiogenesis, the $\alpha_{w} \beta_{3}$ integrin uses the RGD sequence in the tenth fibronectin (III)-like domain of tenascin to bind to extracellular matrix (ECM) (Joshi et al., 1993; Sriramarao et al., 1993), and tenascin in turn has additional binding sites for matrix proteoglycans and heparin. Expression of $\alpha_{w} \beta_{3}$ is ubiquitous in the embryology and development of the vascular system, and expression of members of the tenascin superfamily is similarly widespread in fetal tissue. In adult tissues, although the $\alpha_{v} \beta_{3}$ integrin may be detectable in low amounts (Suzuki et al., 1993), expression of tenascin isoforms is markedly downmodulated within one month of birth (Saga et al., 1991). Under abnormal circumstances, such as in tumorigenesis and wound healing, $\alpha_{w} \beta_{3}$ expression increases significantly (Suzuki et al., 1987; FeldingHabermann and Cheresh, 1993; Juhasz et al., 1993). Thus, detection of these proteins associated with the neovascularization, as we report in the present work, indicates the presence of active angiogenesis in aneurysms.

\section{Materials and Methods}

\section{Reagents and antibodies}

Rabbit anti-human $\alpha_{v} \beta_{3}$ polyclonal antibody R838, was purchased from Chemicon (Temecula, CA). Anti-endothelial nitric oxide synthase (eNOS) monoclonal antibody was purchased from Transduction Laboratories (Lexington, KY). Anti-human tenascin polyclonal antibody EH5B01 and monoclonal antibody TN2 were purchased from GibcoBRL Life Technologies (Gaithersburg, MD). For immunohistochemistry, secondary antibodies and substrates used were: goat anti-rabbit IgG conjugated with horseradish peroxidase (HRP) and diaminobenzidine (DAB) (DA KO Ltd, Copenhagen, Denmark) against R838 and eNOS, and goat anti-rabbit or mouse $\lg G$ antibodies conjugated with alkaline phosphatase and NTB/BCIP (Biorad, Hercules, CA) against EH5B01 or TN2. Other materials used were pepsin (Sigma, St. Louis, MO), Rnase-free DNase I and RNase (Gibco Life Tech., Gaithersburg, MD), RNase inhibitor (RNasin) and EZrTth RNA PCR kit (Perkin Elmer Corp., Norwalk, CT), digoxigenin-11-UTP and anti-digoxigenin antibody conjugated with alkaline phosphatase (Boehrin- ger Manhein Corp., Indianapolis, IN), bio-11-dUTP and streptavidin-biotin alkaline phosphatase complex from ENZO (Farmindale, NY), ultrapure PCR water (Res. Gen., Huntsville, AL). $\alpha_{v}$ and $\beta_{3}$ primers purchased from Gibco Life Tech. Corp. (Gaithersburg, $M D)$, were designed according to the published human av sequences $(22,25)$. The av 5' primer, 5'GACTGTGTGGAAGACAATGTCTG TAAACCC, starts at position 1914 to 1943 of human av cDNA (HAV), and the $\alpha_{w}$ 3' primer, 5'-CCAGCTAAGAGTTGAGTT CCAGCC, from 2195 to 2219 of HAV. The $\beta_{3} 5^{\prime}$ primer, 5'-TTCGACGA GATCATGCA, starts at position 762 to 778 of human $\beta_{3}$ cDNA (HIB3), and the $\beta_{3}$ 3' primer, 5'-AAGGTCCCGTTCCCGTTGTTGCA, from 1462 to 1485 of HIB3.

\section{Immunohistochemistry}

Aortas were obtained from 17 consecutive patients undergoing elective resection of AAA. All patients presented with abdominal aortic aneurysms of $5 \mathrm{~cm}$ in diameter size or greater. None of the patients suffered from rupture of AAA at the time of surgery. The sections of the AAA wall were reviewed macroscopically. Normal control aortic tissues were from 6 organ donors and 1 aorto-occlusive patient (ages average $38 \mathrm{yr} ; 4$ male; 3 female). All tissues were fixed in buffered formalin $(\mathrm{pH} \mathrm{7.4)}$ for 16-24 h.

Histological sectioning and immunohistochemistry were processed as in previous work (Fu et al., 1994). Briefly, aortic paraffin embedded tissue sections (4 $\mu \mathrm{m}$ thick) were dewaxed in xylene and absolute ethanol, inhibited for endogenous peroxidase activity in $0.5 \%$ hydrogen peroxide in absolute methanol (20 $\mathrm{min})$, and blocked with $0.2 \%$ BSA to prevent nonspecific binding. The anti- $\alpha_{w} \beta_{3}$ antibody R838 (1:100) and the anti-tenascin antibodies, EH5B01 (1:50) and TN2 (1:200), were applied to the sections overnight at $4^{\circ} \mathrm{C}$. The anti-eNOS antibodies $(1: 2000)$ were applied to slides following a microwaving step used to enhance antigenicity. Following PBS washes, the sections were exposed to the appropriate secondary antibody for $1 \mathrm{~h}$ at room temperature. After PBS washes the sections were incubated for $10 \mathrm{~min}$ at room temperature with either $D A B$ solution in PBS containing $0.01 \%$ hydrogen peroxide, or NBT/BCIP solution, depending on whether the secondary antibody was respectively, HRP or alkaline phosphatase linked. The sections were finally washed in running tap water, counterstained with hematoxylin for $D A B$ or Nuclear Fast Red for NBT/BCIP, dehydrated in a reversed alcohol-xylene series, and mounted for semi-quantitative morphometric analysis, microscopy and photography. 


\section{Semi-quantitative morphometric analysis of micro- vessels}

Quantitation of microvessel staining by anti-eNOS was carried out by an observer blinded to the source of each specimen, using three criteria: (1) overall impression of staining at $100 \mathrm{X}$ magnification, graded on 1 to 4 scale $(1=$ minimal, $2=$ easily detectable, 3 = moderate, 4 = extensive), (2) the number of capillaries identified in the most densely positive 100 $x$ field, and (3) the number of endothelial cells identified in the most densely positive $400 \times$ field.

\section{Reverse transcription (RT) in situ PCR}

For in situ PCR, we modified Nuovo's method (Nouvo, 1994) and used the EZ rTth RNA PCR kit as follows: after dewaxing and air drying, all sections were digested in $2 \mathrm{mg} / \mathrm{ml}$ pepsin in $0.01 \mathrm{~N} \mathrm{HCl}$ (45 min, room temperature). The pepsin was inactivated by 1 min washes in ultrapure water and absolute ethanol, then the sections were air dried. As a positive control, amplification of tissue genomic DNA was performed by carrying slides directly on PCR cycles without digestion by DNAse I. For detection, the sections were digested by RNase-free DNase I $(400 \mathrm{U} / \mathrm{ml}$ in digestive buffer) overnight at $37^{\circ} \mathrm{C}$. To control for nonspecific binding and background staining, after digestion of DNAse I, the slides were further digested by RNase $H(50 \mathrm{U} / \mathrm{ml}$ in the digestive buffer) for 2 hours at room temperature.

For RT and in situ PCR processes, $50 \mu \mathrm{l}$ of the reagent mixure $(1 \times E Z$ buffer, $200 \mu \mathrm{M}$ of $\mathrm{dNTP}, 15$ $\mu \mathrm{M}$ of -11-dUTP, $2.5 \mathrm{mM}$ of $\mathrm{Mn}(\mathrm{AOc})_{2}$ solution, 0.45 $\mu \mathrm{M} /$ each of $\alpha_{v}$ or $\beta_{3} 5^{\prime}$ and $3^{\prime}$ primers, $2.5 \mathrm{U} / 50 \mu \mathrm{l}$ of rTth DNA Polymerase, and $60 \mathrm{U}$ RNasin) was added to each section and sealed by the In Situ PCR assembly tool (Perkin Elmer Corp., Newark. CT). After incubation for $25 \mathrm{~min}$ at $62^{\circ} \mathrm{C}$ for reverse transcription, the PCR cycles were instituted in an in situ PCR Cycler (Ampligen PCR 1000, Perkin Elmer Corp., Newark, CT) under modified conditions (10 cycles: 94 $\mathrm{C}$ for $60 \mathrm{~s}, 65 \mathrm{C}$ for $60 \mathrm{~s}$ and $72 \mathrm{C}$ for $120 \mathrm{~s}$ ).

To determine the specificity of $\alpha_{v}$ primers, we sampled aliquots of the supernatant on tissue sections immediately following the RT in situ PCR procedure, with or without treating the sections with DNase I. The aliquots were loaded on to $1 \%$ agarose gel and Southern blotted by $\alpha_{v}$ or $\beta_{3}$ probes. These probes (kindly provided by Dr. D. Shinar) were synthesized and labeled with biotin-11-dUTP by PCR amplification of rat $\alpha_{v}$ (RAV 611) or $\beta_{3}$ (RIB 494/3) cDNA, using oligonucleotide primers (shown as above) (Shinar et al., 1993). The membrane was developed using Streptavidine-biotin-alkalinephosphatase and NBT/BCIP.

The sections were chilled at $4^{\circ} \mathrm{C}$ for $20 \mathrm{~min}$, and blocked by $0.2 \%$ BSA in $0.1 \mathrm{SSC}$ solution at $45^{\circ} \mathrm{C}$ for $10 \mathrm{~min}$. Then the slides were incubated in goat anti-digoxigenin antibody conjugated with alkaline phosphatase for $30 \mathrm{~min}$ at room temperature. After 3 washes of Tris buffer $(0.1 \mathrm{M}, \mathrm{pH} 7.5)$, the sections were treated for $10 \mathrm{~min}$ at room temperature with NBT $(2.5 \mu \mathrm{l} / \mathrm{ml}) / \mathrm{BCIP}(2.5 \mu \mathrm{l} / \mathrm{ml})$ in Tris-buffered $\mathrm{CaCl}_{2}$ solution ( $\mathrm{pH}$ 9.5). The development was stopped by washing in running tap water. The sections were counterstained with Nuclear Fast Red, dehydrated and mounted for viewing.

\section{Results}

\section{Microvessels detected by H\&E and anti-eNOS}

Histological sections of aortic aneurysms by $H \& E$ staining showed the presence of several hemorrhagic regions, large numbers of inflammatory cells and numerous microvessels (Figure 1). The inflammatory cells consisted mostly of macrophages and lymphocytes that were associated largely with the microvessels in the adventitia. In addition, some sub-intimal and medial regions showed the presence of neutrophils. By contrast, no inflammatory infiltrates or matrix abnormalities were evident in control aortas (Figure 1D).

Anti-eNOS immunohistochemical staining was employed for two reasons. First, detection of the intracellular eNOS enzyme in AAA and control tissue sections confirmed antigenic integrity of the preserved specimens. Second, anti-eNOS staining served as a marker for microvessel quantitation since the antibody detects the endothelial layer of both small and large microvessels.

In normal aortic wall, positive eNOS staining was seen in the endothelial cell layer of microvessels located in the adventitial vasa vasorum. The number of vessels seen per $100 \times$ field was few, varying from none to 6 . Intact medial smooth muscle cell nuclei were noted in most sections and medial elastin lamellae, as expected, were preserved in all control sections (Figure 2B). In AAA sections positive staining was again seen in the endothelial lining of $\mathrm{mi}-$ crovessels. These vessels, however, were numerous compared to controls. Of the $10-30$ vessels present per high power field $(100 \times)$, more than half were thin-walled capillaries of diameter $<10 \mu \mathrm{m}$ and the remainders were thick-walled microvessels of diameter $>25 \mu \mathrm{m}$ and wall thickness $>10 \mu \mathrm{m}$ (Figures $2 \mathrm{~A}$ and $\mathrm{C}$ ). The capillaries were localized to both the subintimal region and the adventitia, while the thick-walled vessels occurred almost exclusively in the adventitia. Also noted in many sections were dense inflammatory infiltrates surrounding both thick-walled microvessels as well as capillaries. These cells, 

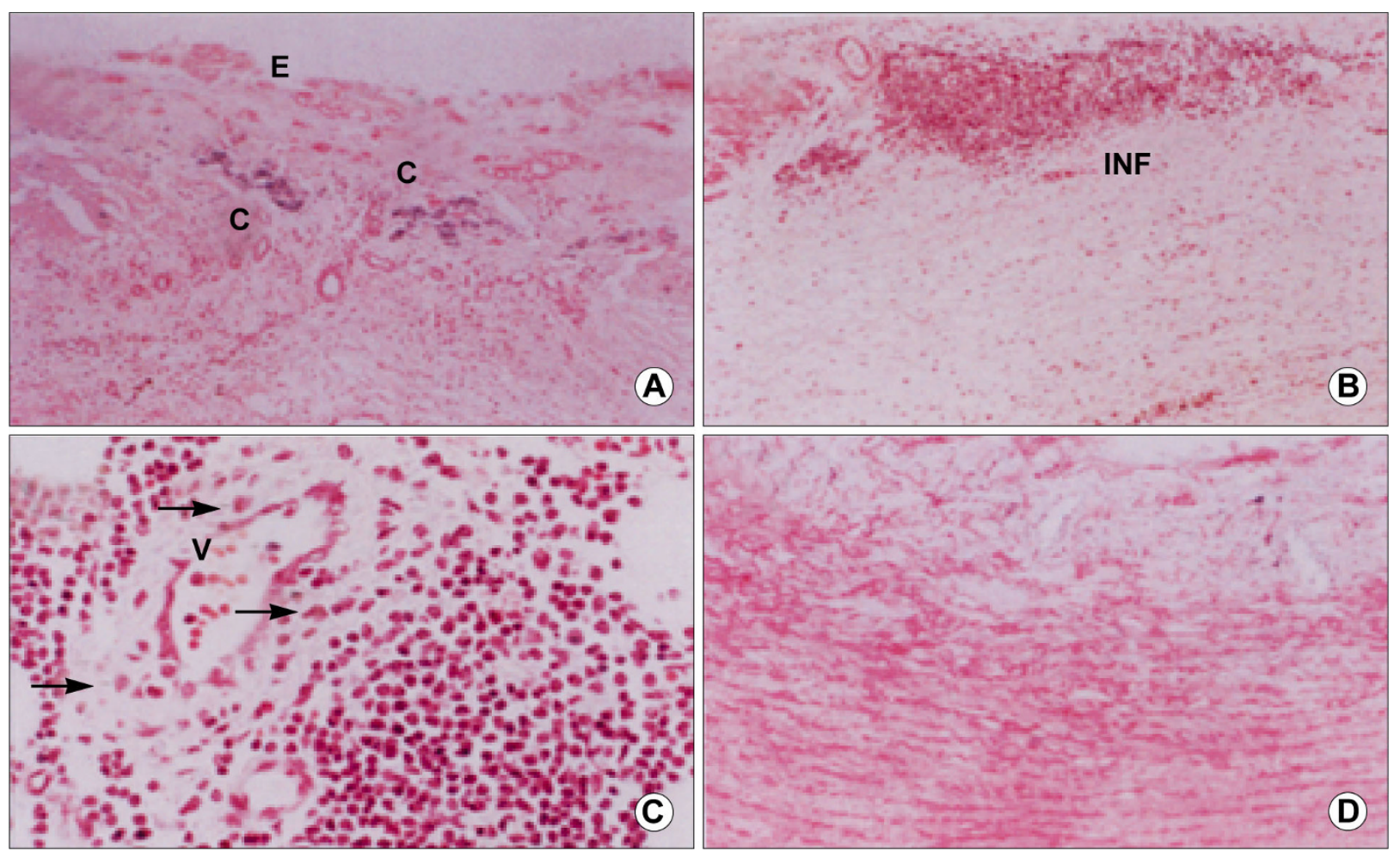

Figure 1. Hematoxylin and Eosin staining. (A) low power view of aneurysm cross-section shows presence of multiple thin-walled capillaries (C) and intact endothelium (E) in the intima and sub-intima (40x). (B) The same section shows the presence of inflammatory infiltrate (INF), thick-walled vessel in the media and adventitia (x40). (C) high power view of aneurysm shows dense accumulation of macrophages (arrows) and lymphocytes associated with a thick-walled vessel (v) (100x); (D) low power view of normal non-aneurysmal aorta shows absence of infiltrates and modest amount of vascularization in the adventitia.

based on their morphology on H\&E staining, were mostly macrophages and lymphocytes. Lastly, elastic lamellae, in contrast to controls, were markedly disrupted and depleted. The results of the anti-eNOS morphometric analysis are shown in Figure 3. The differences between AAA and controls were striking. Mean capillaries were increased 15 -fold per $100 \times$ field $(P<0.001)$, and mean endothelial cells 8 -fold per $400 \times$ field $(P<0.001)$ in 17 AAA specimens by comparison to 7 controls. The general increase in staining for eNOS was highly remarkable in the AAA, although endothelial cells from control patients had approximately equivalent staining reactions on a per cell basis.

\section{Anti- $\alpha_{v} \beta_{3}$, anti-tenascin, and mRNA $\alpha_{v}$ studies}

To determine the specificity of $\alpha_{v}$ primers, we sampled aliquots of the supernatant on tissue sections immediately following the RT in situ PCR procedure, with or without treating the sections with DNase I. Then we blotted the aliquots using our $\alpha_{v}$ probe. As shown in Figure 4, in non-aneurysm tissues a band was detectable only in the absence of DNase I treatment, which is expected because the probe can recognize a segment of non-digested genomic DNA (Figure 4 , lane 1 ). In the presence of DNase I treatment, the band was detectable in aneurysm tissue (Figure 4, lane 2). No band was detectable in either non-aneurysm tissue (Figure 4, lane 3 ) or in aneurysm tissue that had been digested with both DNase I and RNase $H$ (Figure 4, lane 4). These results confirmed that the $\alpha_{v}$ probe specifically recognized the enhanced mRNA expression in aneurysm tissue.

The aortic wall distribution of the $\alpha_{4} \beta_{3}$ antigen is shown in Figure 5. In aneurysmal aortas, an overwhelming majority of microvessels, including capillaries, stained positively for the $\alpha_{w} \beta_{3}$ antigen. However, no staining occurred over the aortic endothelium, the medial and adventitial stroma, and in most large microvessels. A large number of macrophages, particularly those associated with microvessels, also stained positively. No staining was evident in either control aortas or in aneurysms in which R838 was replaced with non-immune rabbit serum as the primary antibody (negative control).

RT in situ PCR of tissue sections of aneurysms revealed co-localization of the $\alpha_{v}$ mRNA with the $\alpha_{w} \beta_{3}$ antigen detected by immunohistochemistry (Figure 5). 


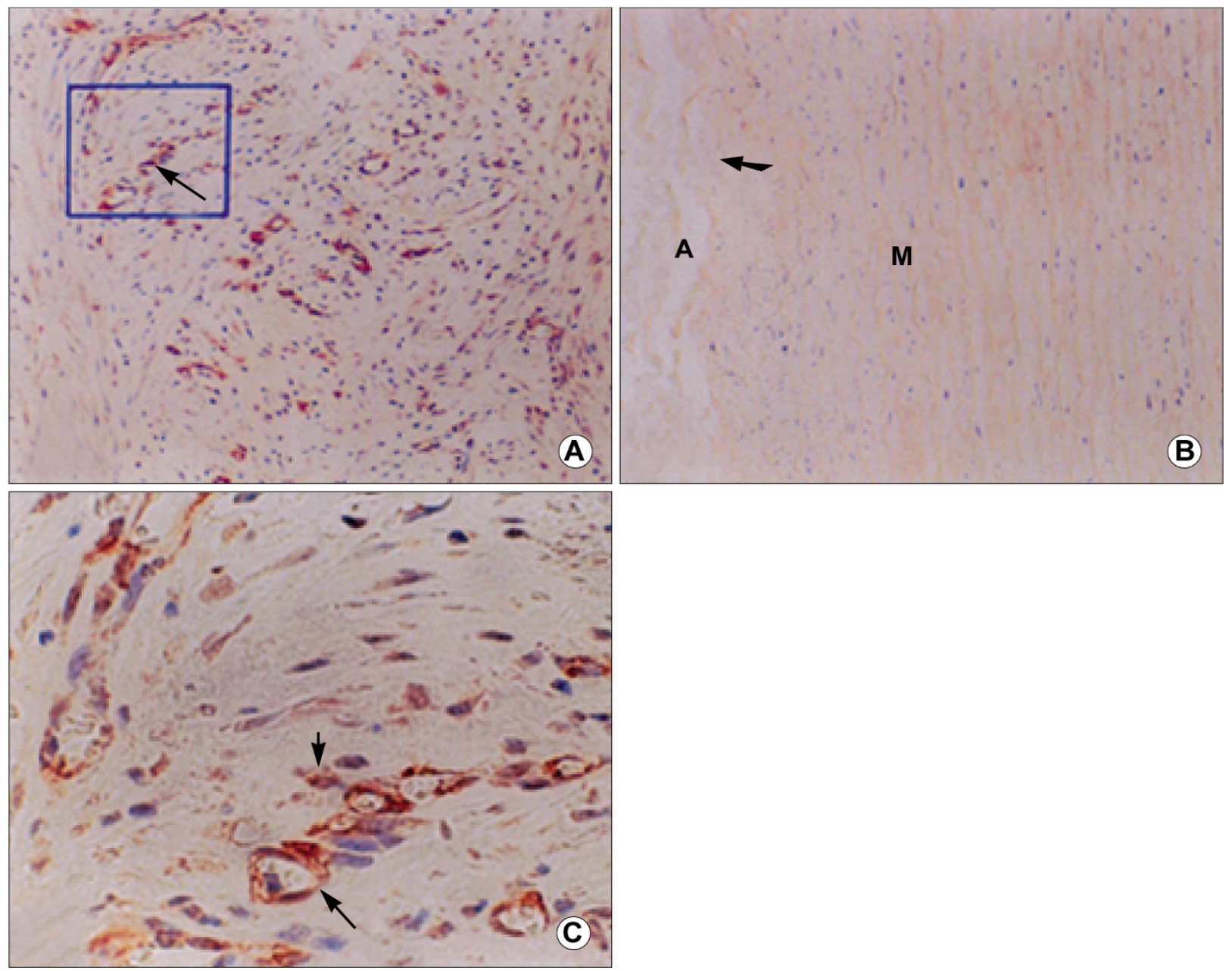

Figure 2. Anti-eNOS immunohistochemical staining (A) AAA wall at $40 \times$ magnification. Most apparent are numerous eNOS-staining microvessels (arrow) outlined in brown stain. They are pictured infiltrating the AAA wall. Greater than 20 distinct lumens are identifiable. There is disarray of the normal regular elastic lamellar architecture (in comparison with B). Although the photomicrograph depicts the expected location for the adventitia/media interface, it is undiscernable. The arrow marks a single capillary, whose endothelial cell is better seen under higher magnification. (see panel C) (B) Non-aneurysmal aortic wall at $40 \times$ magnification. Intact aortic media is free of microvessel invasion and hence dark brown eNOS staining is absent. The adventitia/media interface is clearly visible to the near left (arrow) and loose adventitial tissue (A) is seen to the far left. Long continuous strands of elastic tissue appear translucent, forming a regular orderly pattern within the media (M). Mutiple smooth muscle cells with blue staining nuclei are seen within the aortic media interspersed between elastic lamellae. (C) $100 \times$ magnification of boxed portion of panel A. An endothelial cell (large arrow) outlines a microvessel, staining dark brown with anti-eNOS antibody. Intraluminal translucent red and white blood cells are easily seen at this magnification. Cell nuclei are stained blue but are poorly visualized in endothelial cells that stain positive for eNOS. The tissue stroma contains a single mononuclear inflammatory cell (small arrow).

mRNA staining was much more extensive than antigen staining. Almost all vessels, irrespective of diameter or location, most macrophages, and some lymphocytes, stained positively for $\alpha_{v}$ mRNA. We studied $\beta_{3}$ mRNA in one aneurysm specimen and found a distribution for $\beta_{3}$ mRNA that closely resembled that of the $\alpha_{w}$ mRNA (not shown). PCR products of $\alpha_{w} \beta_{3}$ integrin were not evident in either non-vascularized regions of aneurysms or in nonaneurysmal control aortas (Figure 5). These results prove that both the transcription and the expression of the $\alpha_{v} \beta_{3}$ integrin are upregulated in aneurysm microvessels. The distribution of tenascin staining around microvessels was closely linked to that of $\alpha_{v} \beta_{3}$. Tenascin staining typically was seen circumscribing thick-walled adventitial microvessels (Figure $6)$. Tenascin was also evident in subintimal regions and in areas of medial fibrosis.

\section{Discussion}

Although neovascularization of the aneurysm wall has been previously reported (Koch et al., 1990; Tilson 


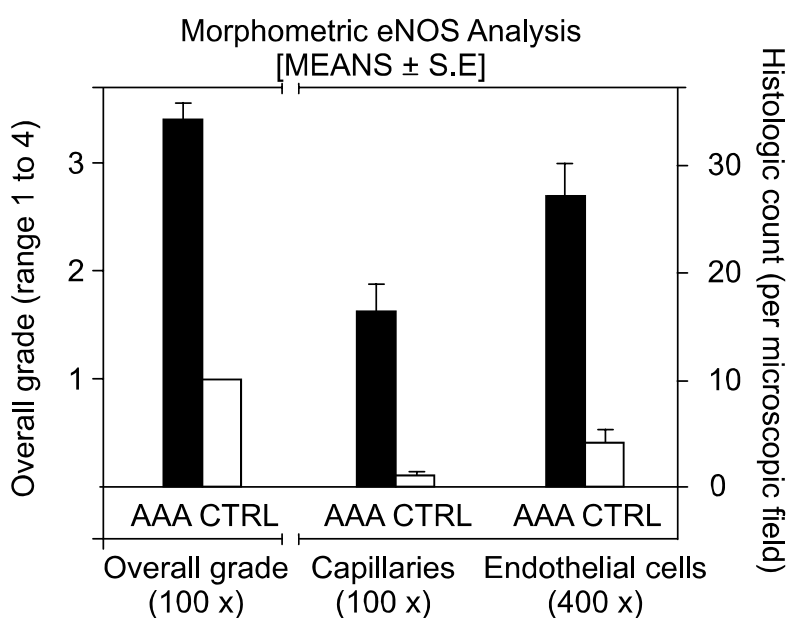

Figure 3. Results of morphometric semi-quantitative analysis of eNOS immunohistochemical studies. Evaluation was carried out using three criteria (see Methods). Mean values are expressed with standard errors. All bars shown were statistically significant at $P<0.001$. Such findings underscore the striking increases in eNOS-staining microvessels observed in the AAA.

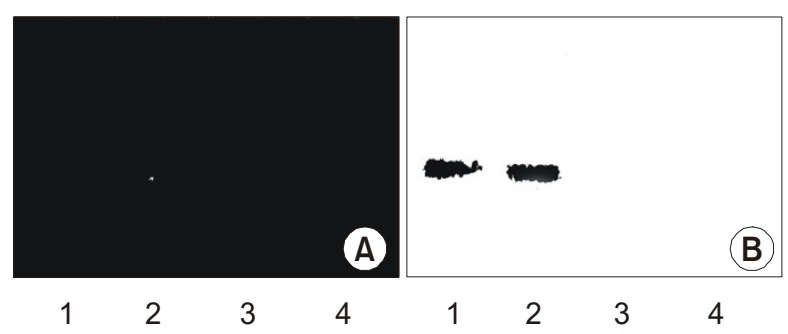

Figure 4. Presence of an amplified product of $308 \mathrm{bp}$ encoding the genomic DNA of av integrin in human aortas and mRNA in AAA tissue. Dnase-untreated human aortas, AAA and normal aortic tissue were used for amplification by RT In situ PCR, using oligonuceotide primers (see Materials and Methods). Amplified fragments were then analyzed on a $1 \%$ agarose gel followed by Southern blotting. (A) Ethidium bromide staining of av-specific PCR-amplified fragments from aortic genomic DNA (lane 1), AAA tissue (lane 2), normal aortic tissue (lane 3) and Dnase- and Rnase-treated tissue (Lane 4). Samples used to generate data in lane 2 were treated with Dnase I in order to exclude the possibility of contamination of samples with genomic DNA. (B) Results of a Southern blotting of cDNA amplified with $\alpha_{v}$-specific oligonucleotide primers. DNA fragments were blotted onto a nitrocellulose membrane and hybridized with a biotin-11-dUTP labeled $\alpha_{v}$-specific probe, detected by streptavidin conjugated with alkaline phosphatase and NBT/BCIP. (see Materials and Methods). Lanes 1-4 same as in (A).

et al., 1990; Brophy et al., 1991; Holmes et al., 1995; Schneiderman et al., 1995) the literature does not clarify whether new vessels appear early or late in the disease, or whether the vasculature undergoes continuous development and remodelling as the aneurysm enlarges. Our findings using an anti-eNOS Ab indicate that all layers of the aneurysm wall are vascularized and display characteristics typical of angiogenesis. Most extensive were thick-walled microvessels that displayed outer-medial to adventitial distribution, and thin-walled capillaries that invested the sub-intima. It is possible therefore, that vessels proliferating in the outer wall also developed transwall capillarization as part of a global neovascularization of the aneurysm. Two angiogenesis markers, namely the $\alpha_{w} \beta_{3}$ integrin and tenascin, were widely detectable in these blood vessels. We conclude from these findings, that vascularization of the aneurysm is extensive and that new blood vessels form in the aneurysm wall as a result of ongoing angiogenesis.

Evidence for angiogenesis was particularly conspicuous in the immunohistochemistry and the RT in situ PCR studies. The $\alpha_{v} \beta_{3}$ integrin, a vascular integrin of the cytoadhesin family (Albelda and Buck, 1990), is located in both the luminal and abluminal surfaces of endothelial cells as well in vascular smooth muscle cells, and has wide-ranging functions including cell adhesion and cell spreading (Albelda and Buck, 1990; Damjanovich et al., 1992; Joshi et al., 1993) and vascular permeability regulation (Tsukada et al., 1995). We confirmed that in thick-walled microvessels, both the endothelial lining as well as the smooth muscle layers stained positively for the integrin. Recently, integrin $\alpha_{w} \beta_{3}$ has been shown to be essential for angiogenesis and a mAb that blocks binding of ligands to $\alpha_{v} \beta_{3}$, promoted tumor regression of angiogenic blood vessels in chick embryos (Brooks et al., 1994). The authors suggest that inhibition of ligand binding to $\alpha_{w} \beta_{3}$ suppresses neovascularization and selectively promotes apoptosis of vascular cells. Interestingly, $\alpha_{w} \beta_{3}$ expressing blood vessels in aneurysms of the present study, were typically associated with macrophages that also expressed both the $\alpha_{v} \beta_{3}$ protein and $\alpha_{v}$ mRNA. The $\alpha_{v} \beta_{3}$ integrin mediates removal of apoptotic cells by macrophages (Brooks et al., 1994; Flora and Gregory, 1994). Since apoptosis co-exists with growing tissue (Brooks et al., 1994), the presence of $\alpha_{v} \beta_{3}$ expressing macrophages in the vicinity of $\alpha_{v} \beta_{3}$ expressing vessels, further signifies that these locations were focal areas of active tissue growth and cell death.

From the experiments, two important results were, first, $\alpha$ mRNA was undetectable in control aortas but strongly detectable in vessels of the aneurysm wall; second, some aneurysm microvessels failed to stain for $\alpha_{w} \beta_{3}$ although they stained positively for $\alpha_{v}$ mRNA. These findings indicate that increased transcription of the $\alpha_{v}$ gene is a significant feature of aneurysm microvessels. Increased transcription of the $\alpha_{v}$ and $\beta_{3}$ genes are co-associated (Shinar et al., 1993), hence, transcription of $\beta_{3}$ mRNA and $\beta_{3}$ expression also increased in these aneurysms. This is indirectly indicated in these experiments, in the increased staining with a polyclonal antibody that recognizes the $\alpha_{4} \beta_{3}$ 

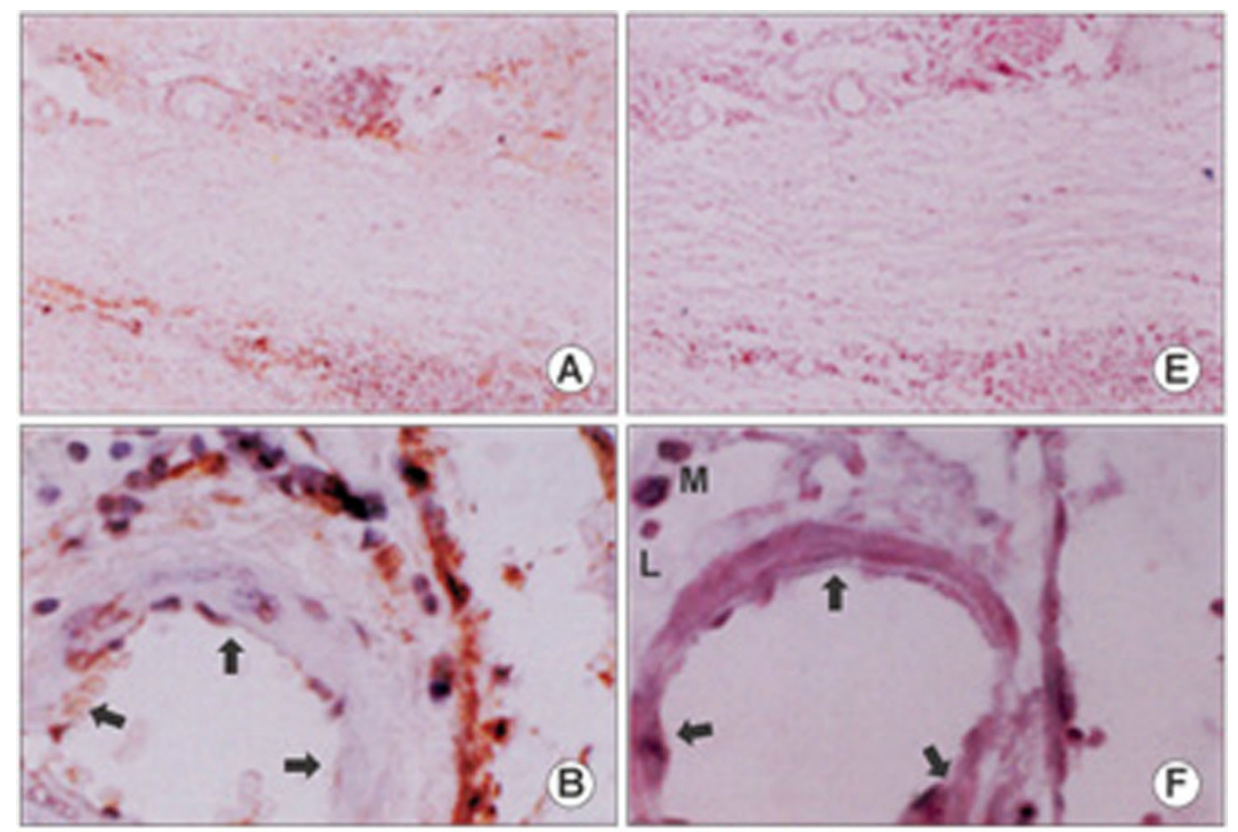

Figure 5. Demonstration of the $\alpha_{v} \beta_{3}$ antigen and the $\alpha v m R N A$ in an aneurysm. $A, B, C, D, G$ show the distribution of the $\alpha_{v} \beta_{3}$ antigen determined by immunohistochemistry. $E, F, H$ show $\alpha_{v}$ mRNA distribution determined by reverse transcription (RT) and in situ PCR. $(A, E)$ Identical $40 x$ views of an aneurysm cross-section. Panel A shows patchy deposition (brown stain) of the anti- $\alpha_{v} \beta_{3}$ immune product over vessels, smooth muscle and inflammatory cells. Panel $\mathrm{E}$ shows extensive blue/purple staining indicative of $\alpha_{v}$ mRNA expression. (B, F) Identical 400x views of a thick-walled microvessel. Arrows point to endothelium. This vessel failed to stain for $\alpha_{v} \beta_{3}$ (panel $B$ ), although it showed endothelial expression of av mRNA (panel F). Expression of $\alpha_{v}$ mRNA in lymphocytes (L) and macrophages (M) is also shown in panel $F$. (C, G) $400 x$ views of a thick-walled vessel (panel $C$ ) and a capillary (c) [panel G] staining for $\alpha_{v} \beta_{3}$. Brown $\alpha_{v} \beta_{3}$ staining is shown in the endothelial $(E)$ and smooth muscle vessel layers (SM) as well as in perivascular macrophages (M) [panel C]. (D, H) $100 x$ view of control aorta shows absence of $\alpha_{v} \beta_{3}$ staining (panel D) and lack of $\alpha_{v}$ mRNA expression (panel $\mathrm{H}$ ).

dimer but not the individual subunits of the integrin (Suzuki et al., 1986). In one case, we determined the distribution of $\beta_{3}$ mRNA by Rt in situ PCR and found this distribution to be similar to that of $\alpha v$ mRNA (data not shown). The mismatch between negative avb3 integrin staining and positive av mRNA expression in some areas indicates that increased $\alpha_{v} \beta_{3}$ expression did not always follow increased gene transcription. This may reconcile the apparent inconsistency between our findings and those recently reported by Cheuk and Cheng (2004) in which no significant difference in RNA transcripts for $\alpha_{v}$ and $\beta_{3}$ were ob- served in AAA vs. control homogenates. Hence, it is possible that increased $\alpha_{4} \beta_{3}$ expression is restricted to specific regions of active angiogenesis.

The immunohistochemical evidence for blood vessel-associated tenascin expression also supports our proposal that angiogenesis was active in these aneurysms. Tenascin is a large oligomeric glycoprotein that appears transiently in the extracellular matrix during tissue modelling and tissue repair (Tremle et al., 1994). Two domains of tenascin, both of which contain the integrin-recognizing arg-gly-asp (RGD) tripeptide sequence, support endothelial cell adhesion 

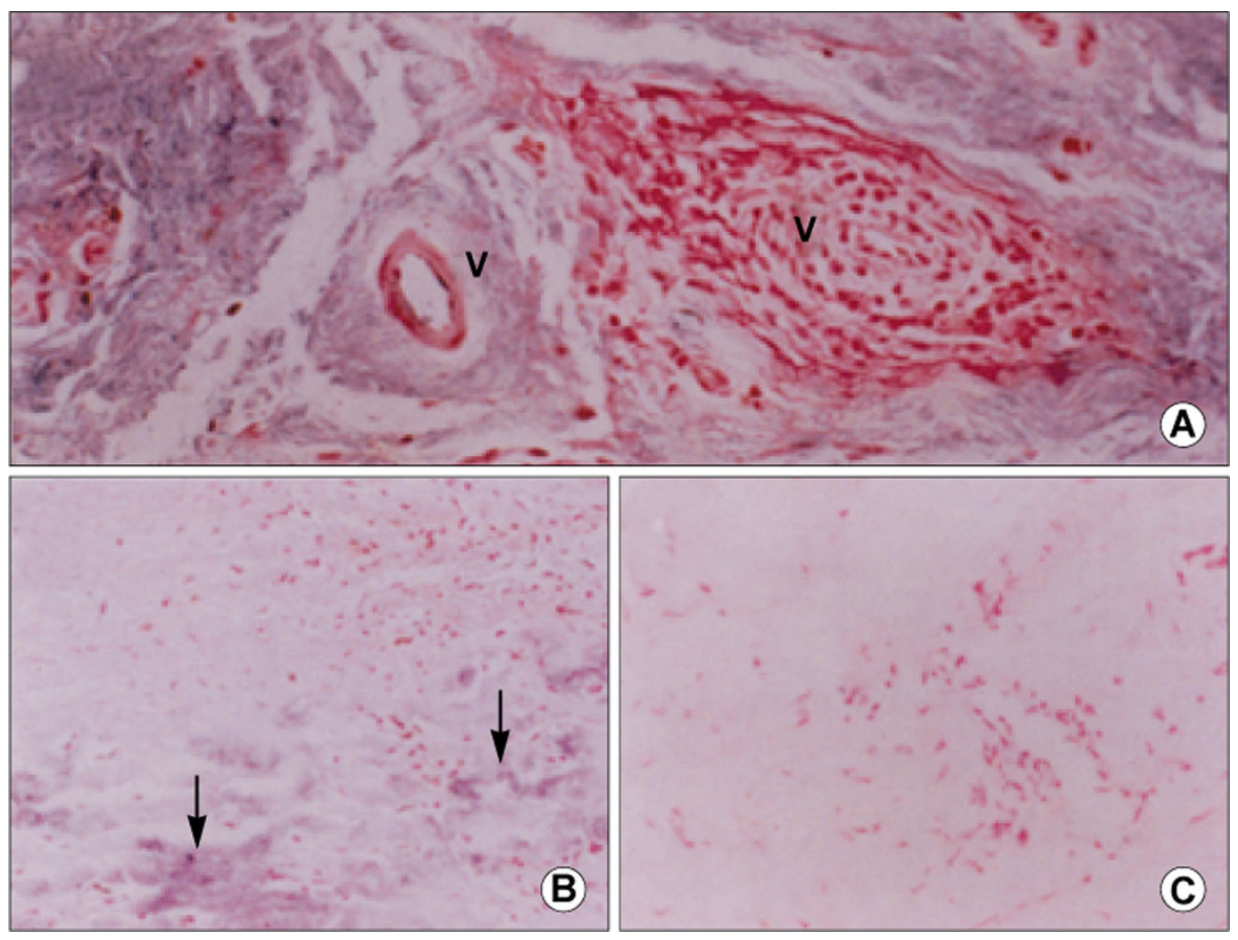

Figure 6. Anti-tenascin immunohistochemical staining. (A) Section of an aneurysm shows distribution of blue/purple anti-tenascin immune product around blood vessel (V). Fast Red counterstaining shows cell distribution in the vessel wall, as well as inflammatory cells in an adjoining vessel. (B) Blue/purple discoloration shows tenascin distribution in the subintima (arrows). (C) Control aorta shows no staining for tenascin.

(Joshi et al., 1993). One of these, the third fibronectin-III domain, also supports cell spreading (Sriramarao et al., 1993). The involvement of endothelial integrins and in particular, the $\alpha_{w} \beta_{3}$ integrin is indicated in that endothelial adhesion to tenascin is RGD dependent (Joshi et al., 1993) and is partly inhibited by anti- $\alpha_{w} \beta_{3}$ antibodies (Sriramarao et al., 1993). The ability of endothelial cells to adhere to tenascin (Joshi et al., 1993) suggests that endothelial tenascin receptors play a supportive role for establishing blood vessel structure during angiogenesis. The circumscribed perivascular tenascin expression seen here, is consistent with this notion and suggests that that tenascin provides a cylindrical template for vessel tube formation. Addition of tenascin to a fibronectin-based substratum upregulates the synthesis of three important matrix metalloproteinases in fibroblasts: collagenase (MMP-1), stromelysin (MMP-3), and gelatinase-B (MMP-9) (Newman et al., 1994; Newman et al., 1994). We have reported that these three matrix metalloproteinases are present in significantly higher levels in aneurysms than in control aortic specimens (Newman et al., 1994). Tenascin may promote aneurysm progression by inducing these metalloproteinases in endothelial cells of new blood vessels as well as in mesenchymal cells.

Using anti-eNOS $\mathrm{Ab}$ directed against endothelial cells, we observed marked increases in neo-vessel number in the aneurysm wall. Although no conclusions can be made concerning the possible role of eNOS or nitric oxide in aneurysm formation from our study, Kuhlencordt et al. have suggested that alterations in eNOS expression play a role in the formation of AAA (2001). Furthermore, we have shown that the nitric oxide by-product, nitrite, is capable of invoking deleterious changes in connective tissue proteins such as collagen and elastin (Paik et al., 2001). Since levels of nitrogen oxide gases are high in tobacco smoke and cigarette smoking is a strong environmental risk factor for AAA disease (Lederle et al., 2003), future studies will be directed at clarifying the role of nitric oxide and its by-products in the formation of AAA.

Finally, our finding that angiogenesis is active in the mature aortic aneurysm, suggests that a potential intervention site exists for pharmacologically reducing the rate of aneurysm enlargement or preventing the disease in genetically susceptible individuals. The observation by Brooks et al. (1994) that the angiogenesis in tumorigenesis can be arrested by antibody to $\alpha_{v} \beta_{3}$ suggests that anti-angiogenic therapy might be considered among other novel interventions that are presently under investigation, based on recent developments in our understanding of aneurysm pathogenesis.

\section{Acknowledgement}

This work was supported by NIH grant HL36024 to JB, the Aneurysm Information Project at St.Luke's/ 
Roosevelt Hospital Center, with NIH grant R01 HL64334-4 to MDT.

\section{References}

Albelda SM, Buck CA. Integrin and other cell adhesion molecules. FASEB J 1990;4:2868

Brooks PC, Clark RAF, Cheresh DA. Requirement of vascular integrin $\alpha_{v} \beta_{3}$ for angiogenesis. Science 1994a;264: 569-71

Brooks PC, Montgomery AM, Rosenfeld MR, Reisfeld A, Hu T, Klier $G$, Cheresh DA. Integrin $\alpha_{\vee} \beta_{3}$ antagonists promote tumor regression by inducing apoptosis of angiogenic blood vessels. Cell. 1994b;79:1157-64

Brophy CM, Smith GJW, Tilson MD. Pathology of Nonspecific Abdominal Aortic Aneurysm Disease. In "Current therapy in vascular surgery" (second edition) (Ernst CB, Stanley JC), pp. 238-41. B.C. Decker Inc, Philadelphia. 1991a

Brophy CM, Reilly JM, Smith GJW, Tilson MD. The role of inflammation in nonspecific abdominal aortic aneurysm disease. Ann Vasc Surg 1991b;5:229-33

Cheuk BLY, Cheng SWK. Differential expression of integrin a5B1 in human abdominal aortic aneurysm and healthy aortic tissues and its significance in pathogenesis. J Surg Res 2004;118:176-82

Damjanovich L, Albelda SM, Mette SA, Buck CA. Distribution of integrin cell adhesion receptors in normal and malignant lung tissue. Am J Respir Cell Mol Biol 1992;6: 197-206

Felding-Habermann B, Cheresh DA. Vitronectin and its receptors. Curr Opin Cell Biol 1993;5:864-8

Flora PK, Gregory CD. Recognition of apoptotic cells by human macrophages: inhibition by a monocyte/macrophagespecific monoclonal antibody. Eur J Immunol 1994;24:262532

Fu CZ, Jasani B, Vujanic GM, Leadbeatter S, Berry PJ, Knight $\mathrm{BH}$. The immunocytochemical demonstration of a relative lack of nerve fibres in the atrioventricular node and bundle of $\mathrm{His}$ in the sudden infant death syndrome (SIDS). Forensic Sci Int 1994;66:175-85

Herron GS, Unemori E, Wong M. Connective tissue proteinases and inhibitors in abdominal aortic aneurysms. Arteriosclerosis and Thrombosis 1991;11:1667-77

Holmes D. R, Liao S, Parks WC, Thompson RW. Medial neovascularization in aneurysm: A histopathologic marker of aneurysmal degeneration with pathophysiological implications. J Vasc Surg 1995;21:761-72

Hynes RO. Integrin: A family of cell surface receptor. Cell 1987;48:549-54

Irizarry E, Newman KM, Gandhi RH, Nackman GB, Halpern $V$, Wishner S, Scholes JV, Tilson MD. Demonstration of interstitial collagenase in abdominal aortic aneurysm disease. J Surg Res 1993;54:571-4

Joshi P, Chung CY, Aukhil I, Erickson HP. Endothelial cells adhere to the RGD domain and the fibrinogenAlike terminal knob of tenascin. J Cell Sci 1993;196:389-400

Juhasz I, Murphy GF, Yan HC, Herlyn M, Albelda SM. Regulation of extracellular matrix proteins and integrin cell substratum adhesion receptors on epithelium during cutaneous human wound healing in vivo. Am J Pathol 1993; 143:1458-69

Koch AE, Haines GK, Rizzo RJ, Radosevich JA, Pope RM, Robinson PG, Pearce WH. Human Abdominal Aortic Aneurysms: Immunophenotypic analysis suggesting an immunemediated response. Am J Pathol 1990;137:1199-219

Kuhlencordt PJ, Gyurko R, Han F, Scherrer-Crosbie M, Aretz TH, Hajjar R, Picard M, Huang PL. Accelerated atherosclerosis, aortic aneurysm formation, ischemic heart disease in apolipoprotein E/endothelial nitric oxide synthase doubleknockout mice. Circulation 2001;104:448-54

Lederle FA, Nelson DB, Joseph AM. Smokers' relative risk for aortic aneurysm compared with other smoking-related diseases: a systematic review. J Vasc Surg 2003;38:329-34

Newman K.M, Ogata Y. Malon A. M. Irizarry, Gandhi E. R. $H$, Nagase $H$. and M.D.Tilson. Identification of matrix metalloproteinases 3 (stromelysin-1) and 9 (gelatinase B) in abdominal aortic aneurysm. Arteriosclerosis and Thrombosis 1994a;14:1315-20

Newman KM, Malon AM. Shin RD. Scholes JV. Ramey WG, Tilson MD. Matrix metalloproteinases in abdominal aortic aneurysm: Characterization, Purification, their possible sources. Connective Tissue Res 1994b;30:265-76

Nuovo GJ. PCR in situ hybridization protocols and applications (second edition). pp:1-416. Raven Press, New York. 1994

Paik DC, Dillon J, Galicia E, Tilson MD. The nitrite/collagen reaction: non-enzymatic nitration as a model system for age-related damage. Con Tis Res 2001;42:111-22

Saga $Y$, Tsukamoto $T$, Jing N, Kusakabe M, Sakakura T. Murine tenascin: cDNA coning, structure and temporal expression of isoforms. Gene 1191;104:177-85

Schneiderman J, Bordin GM, Engelberg I, Adar R, Selffert $D$, Thinnes T, Bernstein EF, Dilley RB, Loskutoff DJ. Expression of Fibrinolytic Genes in atherosclerotic abdominal aortic aneurysm wall: a possible Mechanism for aneurysm expansion. J Clin Invest 1995;96:639-45

Shinar DM, Schmidt A, Halperin D, Rodan GA. Weinred M. Expression of $\alpha_{v}$ and $\beta_{3}$ integrin subunits in rat osteoclasts in situ. J Bone Miner Res 1993;8:403-14

Sriramarao P, Mendler M, Bourbon MA. Endothelial cell attachment and spreading on human tenascin is mediated by $\alpha_{2} \beta_{1}$ and $\alpha_{v} \beta_{3}$ integrins. J Cell Sci 1993;195:1001-12

Suzuki S, Argraves WS, Pytella R, Arai H, Krusius T, Pierschbacher MD, Rouslahti E. CDNA and amino acid sequence of the cell adhesion protein receptor recognixing vitronectin reveal a transmembrane domain and homologies with other adhsion protein receptor. Proc Natl Acad Sci USA 1986;83:8614-8

Suzuki S, Argraves WS, Arai H, Languino LR, Pierschbacher $M D$, Rouslati $E$. Amino acid sequence of the vitronectin receptor a subunit and comparative expression of adhesion receptor mRNAs. J Biol Chem 1987;202:14080-5 
Suzuki S, Takahashi T, Nakamura S, Koike K, Ariyoshi Y, Takahashi T, Ueda R. Alteration of integrin expression in human lung cancer. Jpn J Cancer Res 1993;84:168-74

Tilson MD, Elefteriades J, Brophy CM. Tensile strength and collagen in abdominal aortic aneurysm disease. In "The cause and management of aneurysms" (R. M. Greenhalgh, Mannick JA, Powell JT), pp.97-104. W.B. Saunders Company, London. 1990
Tremle $P$, Chiquet-Ehrismann $R$, Werb $Z$. The extracellular matrix ligands fibronectin and tenascin collaborate in regulating collagenase gene expression in fibroblasts. Mol Biol Cell 1994;5:439-53

Tsukada $\mathrm{H}$, Ying $\mathrm{X}, \mathrm{Fu} \mathrm{CZ}$, Ishikawa S, McKeownALongo $P$, Albelda S, Bhattacharya S, Bray BA, Bhattacharya J. Ligation of the endothelial avb3 integrin increases capillary hydraulic conductivity of rat lung. Circ Res 1995;77:651-9 